\title{
POTENTIALITIES OF YALE ASTROMETRIC MATERIALS
}

\author{
E. D. HOFFLEIT
}

Yale University Observatory, New Haven, Conn., U.S.A.

\begin{abstract}
A brief summary is given of the plate materials and results obtained at Yale since the advent of photographic astrometry. The distributions of plates not yet measured or reduced are indicated and possible supplementary programmes for their effective utilization are discussed. Interest centres chiefly in the following areas:

(1) The unmeasured parallax plates.

(2) Reduction of the $-60^{\circ}$ to $-70^{\circ}$ zone catalogue.

(3) The El Leoncito programmes for the determination of both positions and proper motions relative to faint galaxies.

(4) The feasibility of a search for intrinsically faint nearby stars with the Yale 40-in. at Cerro Tololo and the 20-in. double astrograph at El Leoncito.
\end{abstract}

The majority of photographic plates at the Yale Observatory stem from programs initiated by Frank Schlesinger (1871-1943, known as the 'Father of Modern Astrometry') and supplemented by the interests and energies of Dirk Brouwer (1902-1966). These comprise plates for stellar parallax, zone catalogues of positions and proper motions, as well as planet and asteroid programs planned for ascertaining corrections to star catalogues, and polar plates taken with the Loomis 15-in. polar telescope for investigating the motions of the Earth's axis. Under Dr Brouwer's direction several doctoral theses had been written based on the solar system observations (e.g. Pierce, 1971). To my knowledge no further investigations with these materials are contemplated. My present survey is limited to plates for parallax and proper motion programs.

\section{Parallaxes with the 26-in. Refractor}

Until W. L. Elkin's (1855-1935) retirement from the Directorship in 1910, Yale was among the last of the strongholds adhering to the classical visual technique (split objective heliometer) with which Bessel in 1838 determined the first accurate published parallax. Frank Schlesinger came to Yale in 1920 and the Observatory quickly became recognized as the foremost center for stellar parallax. The momentum of his achievements spurred his successor, Brouwer, with the devoted help of Louise Jenkins and Donald Kimball, to pursue stellar parallaxes right up to the present. The acquisition of parallax plates at Yale ceased in 1963 with the transfer of the 26-in. refractor to Mount Stromlo. After his recent retirement, Kimball has submitted his final list of some 20 parallaxes for publication. This marks the end of a long and distinguished photographic era during which Yale determined some 2100 parallaxes, mainly of southern stars.

However, the reductions of accumulated parallax plates are by no means complete. There remain nearly 6700 parallax plates taken between 1928 and 1963 still unmeasured. While many were intended for improving earlier results, there are available 
from 15 to 43 plates each on 59 stars for which no parallax at all has been determined, and on 53 for which only one determination at some other observatory is available. For nearly 300 stars additional plates had been taken for investigating variable proper motions. Many of the stars in these three groups are Luyten high proper motion stars. This constitutes potential material for mass ratios of nearby Population II stars for which astrophysicists have expressed an urgent need. Indeed, for 23 more stars with already well determined parallaxes, from 10 to 56 additional plates spanning 5 to 32 years had been taken for eventual mass ratio determinations. Although personnel and funding for computer time for this work are sadly lacking, the U.S. Naval Observatory has proffered help in permitting the use of its semi-automatic measuring engine for the plate measurements, and $\mathrm{Dr} \mathrm{L}$. Auer is preparing computer programs for their reduction.

The 26-in. refractor was also used for other programs, both astrometric and photometric. Among the former, as Dr Klemola has pointed out to me, are plates taken for the determination of proper motions of 22 RR Lyrae stars with median magnitudes between 9.5 and 14.0. From two to four plates each had been taken about 1928 and again in 1944-45 on the following stars: SW Aqr, S Ara, X Ari, RV Cap, TX Car, EE Car, AT Cen, RR Cet, RT Dor, RV Dor, RX Eri, SV Hya, RV Leo, V Lep, RZ Lib, T Men, V Men, ST Oph, RV Phe, SS Tau, VX Vel, and ST Vir.

\section{The Zone Catalogues}

Since 1920 the Yale Observatory has published catalogues of the positions and proper motions of approximately a quarter of a million stars mostly brighter than 11 th visual magnitude between declinations $+85^{\circ}$ to $+90^{\circ},+50^{\circ}$ to $+60^{\circ},+30^{\circ}$ to $-50^{\circ}$, and $-70^{\circ}$ to $-90^{\circ}$ (the last only provisional). These results are published in most of the volumes of the Transactions of the Yale University Observatory from Vol. 3 through Vol. 31. All north of $-30^{\circ}$ are largely the achievement Dr Ida Barney who had been Schlesinger's able assistant from the beginning and who carried on the work until her retirement in 1959 . Only the zone from $-60^{\circ}$ to $-70^{\circ}$ has not yet been reduced. The plates for this zone had been measured under the direction of Dr Arnold Klemola in 1965. The former Army Map Service, who financed all of the Yale Zone Catalogue work for the zones south of $-30^{\circ}$, had volunteered to carry out the reductions of this particular zone. All of Klemola's measurements as well as necessary auxiliary reduction data on punched cards were transmitted to the Army Map Service in Washington. However, they did very little work on this project, and last year all of the raw materials were returned to Yale. Reductions must now await adequate funding for programmers and computer time as well as for putting now warped cards onto tapes.

\section{Proper Motions Relative to Faint Galaxies}

This project for extending the Lick program to the South Pole had been initiated 
during the regime of the late Dirk Brouwer. It has become the province of Dr Wesselink who reports on the completion of the first epoch plates in his paper later in this Symposium. While there is by no means unanimous agreement on whether the second epoch plates should begin after a lapse of 10 or of 20 years, the problem is unfortunately resolved for the present by the lack of support of the YaleColumbia Southern Station beyond 1973. This same lack of support militates as well against the initiation of other contemplated Yale projects for the 20-in. southern astrograph at El Leoncito.

\section{Proposed Search for Faint Nearby Stars}

Two recent independent investigations (Murray and Sanduleak, 1972; Weistrop, 1972) suggest that there are many more faint nearby red dwarfs than have already been found, or than are implied by the luminosity function derived by Luyten from his discoveries of high proper motion stars. The vast majority of the undiscovered stars would presumably be members of the disc population, with circular motions too nearly parallel to that of the sun to be detected in a selective survey for large proper motions. It is not my purpose here to discuss how valid is the evidence for their existence; rather I should like to consider the feasibility of a search for such stars with available Yale equipment should they indeed exist.

Several factors are considered: (1) The number of such stars to be expected in the areas of plates taken with the Yale telescopes; (2) The total number of stars in the field among which the parallax stars are to be detected; (3) The average number of high proper motion stars expected in the field; (4) The time factors necessary for making the search; (5) and most importantly, the potential accuracy of measurements for this purpose.

Table I summarizes the expected frequencies for plates reaching limiting visual magnitude 18. The numbers of faint red dwarfs within 20,10 and 5 parsecs are based on Weistrop's luminosity function for the disc population. The number of high proper motion stars is inferred from Luyten's (1971) published results of his blinking 48-in. Palomar-Schmidt plates. The numbers of field stars are interpolated from the Searsvan Rhijn (1925) star counts on Mount Wilson plates.

TABLE I

Expected numbers of stars per sq deg

\begin{tabular}{lc}
\hline Category & No. \\
\hline Parallaxes $\geqslant 0.050$ & 1.2 \\
Parallaxes $\geqslant 0.100$ & 0.2 \\
Parallaxes $\geqslant 0.200$ & 0.02 \\
Proper motions $\geqslant 0$ ".100 & 20 \\
Field stars at pole of Galaxy & 660 \\
Field stars at pole of Ecliptic & 3400
\end{tabular}


Two telescopes might be available for the search. The southern El Leoncito 20-in. astrograph has a scale of $55^{\prime \prime} \mathrm{mm}^{-1}$ and takes 17-in. square plates covering $6^{\circ} \times 6^{\circ}$. The Yale 40 -in. reflector with a scale of $20^{\prime \prime} \mathrm{mm}^{-1}, 5 \times 7$ in. plates covers 0.7 square degrees. It is now being re-located at Cerro Tololo. At least two parallax search plates would be taken at each of three consecutive epochs at intervals of approximately six months (the third in order to eliminate proper motion), at times of largest possible positive and negative parallax factors. If the plates are measured to an accuracy of one micron, the best accuracy that can be expected from a parallax determination is about 0.05 on El Leoncito plates, and 0".02 on plates taken with the 40 -in. Thus the El Leoncito plates would be adequate for the detection of stars at best to $10 \mathrm{pc}$ and probably not beyond $5 \mathrm{pc}$ (one to 7 parallax stars per region photographed). The 40 -in. could be of marginal value almost out to the desired 20 pc (30 to 40 parallax stars in an aggregate area equal to that of an El Leoncito plate.

With the Yale Mann measuring engine whose only automatic feature is to record measured coordinates on punch cards, the measurements of all of the stars on an El Leoncito plate reaching $18 \mathrm{mag}$. would require well over $900 \mathrm{~h}$ for the pole of the ecliptic and 200 at the galactic pole. The same total number of stars measured on the small plates taken with the 40-in. telescope would take longer because of more plate handling. With automatic or semi-automatic engines such as the GALAXY or the U.S. Naval Observatory machines, the measuring time would be reduced by approximately $75 \%$ and all of the stars in a $36 \mathrm{sq}$ deg area at the galactic pole could be measured in little over a week. Moreover, with automatic image centering the setting errors might be reduced to half a micron.

If only one star in nearly 700 at the pole of the galaxy is expected to have a large parallax, one wishes these stars could be discovered by means of the blink comparator rather than through prolonged precision measurements. Luyten's experience during his Bruce Proper Motion Survey indicated that with plates having a scale of $60^{\prime \prime} \mathrm{mm}^{-1}$ and a time span of 10 years he could detect stars with proper motions of 0 " 100 , corresponding to image displacements of $0.02 \mathrm{~mm}$ between the two plates being blinked. This would imply that with the El Leoncito plates only stars within 2 pc could be found by this technique. On the 40 -in. plates the limiting parallax discoverable would be close to 0.20 , whence an average of 50 plates might have to be blinked to find just one nearby star - a futile effort for the purpose in hand.

Parallax stars indicated by precise coordinate measurements of a limited number of plates, as described above, should for the most part be considered only as good suspects whose parallaxes would ultimately have to be established by more extensive series of standard parallax observations.

\section{References}

Luyten, W. J.: 1971, Proper Motion Survey with the Forty-Eight Inch Schmidt Telescope, No. XXVII, Univ. Minnesota.

Murray, C. A. and Sanduleak, N.: 1972, Monthly Notices Roy. Astron. Soc. 157, 275.

Pierce, D.: 1971, Astron. J. 76, 177. 
Sears, F. H., van Rhijn, J. P., Joyner, M. C., and Richmond, M. L.: 1925, Astrophys. J. 62, 320. Weistrop, D.: 1972, Astron. J. 77, 366 and 849.

\section{DISCUSSION}

Luyten: I feel I must take issue with the last part of the paper: if there really is 1 star per sq deg nearer than $20 \mathrm{pc}$ and brighter than $18^{m}$, then nearer than $5 \mathrm{pc}$ (where they would be $3^{m}$ brighter) there should be 1000 stars brighter than $18^{m}$ and this I just can't accept.

As to Miss Weistrop's result: some twelve years ago I published an analysis made near the South Galactic Pole on three-image plates which Haro and I took with the Palomar 48-in. and I found a great dearth of $M$ stars and of course faint stars nearer than $20 \mathrm{pc}$ should be $M$ dwarfs: but at that time this was an unpopular conclusion and it is now - so it was simply swept under the rug.

Murray: I don't want to be forced into a position about these stars. All I did was measure proper motions of 21 stars, and from these it appeared that the small dispersion and fairly large mean motion indicated that they were relatively nearby and had a small velocity dispersion. Arguments about the local mass density are based on gross extrapolation, and may well be wrong. If there are a large number of young objects around, then they may well appear in groups and perhaps in the north polar region we have hit such a group. 